\title{
Enhanced Skin Adhesive Property of Hydrophobically- Modified Poly(Vinyl Alcohol) Films
}

\section{Supporting Information}

\author{
Xi Chen ${ }^{\dagger} \star \star$, Tetsushi Taguchi*†, \\ ${ }^{\dagger}$ Graduate School of Pure and Applied Sciences, University of Tsukuba, 1-1-1 Tennodai, Tsukuba, \\ Ibaraki 305-8577, Japan \\ †iomaterials Field, Research Center for Functional Materials, National Institute for Materials \\ Science, 1-1 Namiki, Tsukuba, Ibaraki 305-0044, Japan
}

First author

Tel: +81-29-851-8746

Fax: +81-29-860-4752

E-mail: CHEN.Xi2@nims.go.jp

*Corresponding author

Tel: +81-29-851-4498

Fax: +81-29-860-4752

E-mail: TAGUCHI.Tetsushi@nims.go.jp 


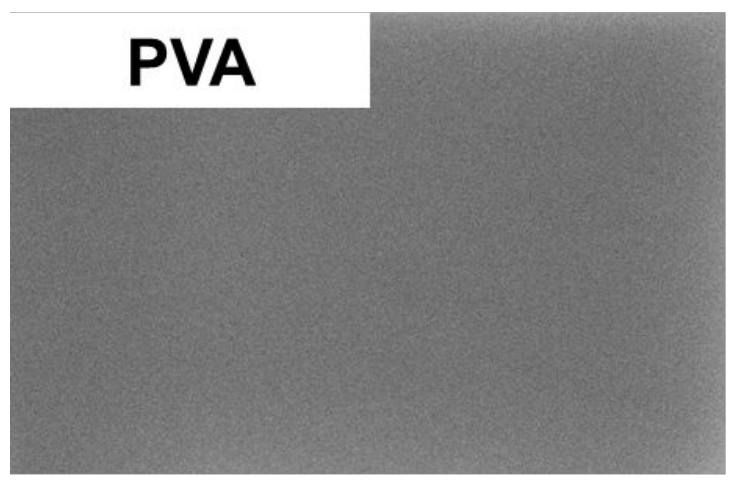

\section{C3-PVA}

\section{C6-PVA}

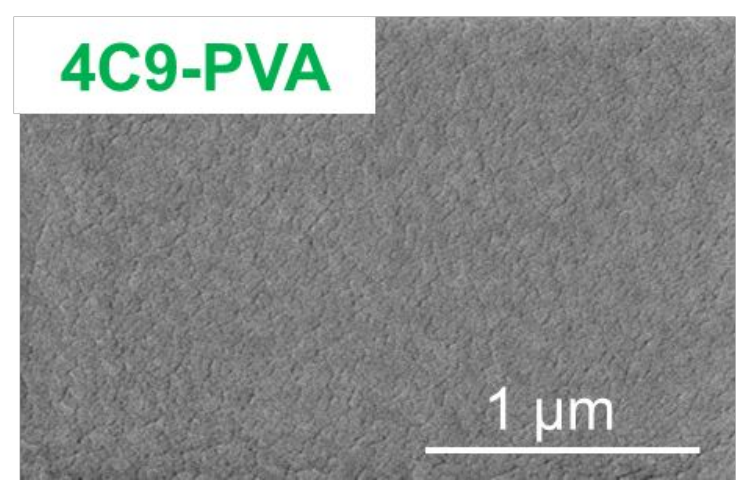

Figure S1. The SEM images of the surface of PVA and hm-PVA films. 